\title{
AN OVERVIEW OF KEY TECHNOLOGIES FOR THE NEXT GENERATION NETWORKS
}

\author{
Howard Green ${ }^{1}$, Pierpaolo Ghiggino ${ }^{1}$ \\ 'Marconi Communications, Stoneleigh House, New Century Park, Coventry, CV3 IHJ, UK
}

\begin{abstract}
This article presents a view of some of the key new technologies that are likely to play a significant role in the deployment of the next generation network architectures necessary to support the needs of operators.
\end{abstract}

\section{OPTICAL TRANSPORT NETWORKS (OTN)}

Most large operator networks today have a substantial transport layer of SDH/SONET devices, with fibre bottlenecks and shortages being avoided by the use of WDM point-point systems. As networks evolve to carry mostly data, there is scope to reduce network complexity by "de-layering". Nonetheless, we believe that there continues to be a clear role for a transport layer ${ }^{1}$, providing flexible protected transport between network service nodes, carrying customer leased line services, and supporting the scalability of higher network layers. In the short term, many operators have substantial overcapacity in their core transport networks. Nonetheless, this capacity will be exhausted. In a three to five year time frame, transport networks will need increased capacity. Moreover, operators will wish to use this opportunity to simplify their networks and reduce operational costs.

In this context, we believe that there will be a substantial integration of the SDH and WDM sub-layers in the transport network. The ITU OTN standards (e.g. G.872, G.709 "Digital Wrapper", G.798, G.959.1) provide for a consistent hierarchy of bearers, multiplexing and trail overhead, allowing the transport of all kinds of signal formats up to and including services (e.g. Fibre Channel, DVI

\footnotetext{
${ }^{1}$ In particular in the regional and core parts of networks. There is a tendency towards the "collapsing " of transport, aggregation and switching at the edge of the network.
} 
video, low latency GigE and 10GigE) which are currently transported as "wavelength services". This extension of standard transport principles will also allow the deployment of $40 \mathrm{~Gb} / \mathrm{s}$ line rates in a consistent fashion when demand requires and the technology is cost-effective. It allows for standardized intervendor and inter-operator connectivity, and supports consistently a standard set of features including dedicated and shared protection and virtual concatenation.

Thus we believe that the dominant switching paradigm in transport networks for the foreseeable future will be "opaque" O-E-O switching. There will be a role for transparency, in particular to provide "optical bypass" of intervening traffic nodes to save the cost of transponders. WDM technology will be progressively integrated into OTN-capable transport platforms, starting with coloured interfaces and later allowing integration of filters, "managed optical patch panel" etc. In this context we believe that WDM will increasingly be thought of as a network and interface technology, rather than a network layer in its own right.

\section{AUTOMATED SWITCHED TRANSPORT NETWORKS (ASTN)}

Today there is a significant difference in operating data networks and transport networks. While data networks tend to use distributed protocols for switching and routing, transport networks are centrally managed.

Part of this difference just reflects the differing roles of these network layers.

At the network edge in the service layer, network elements must deal with many fine-grain service requests from very large numbers of users. Thus by necessity control must be distributed and respond in real time. By contrast, at the network core in the transport layer, long term planning is unavoidable. Fibre must be laid and capacity hired. Thus trails are bound to be of longer lifetime and higher capacity.

However, these network layers have historically been designed quite separately. They have different control technologies, operating procedures and user interfaces.

However we believe that these elements (bandwidth planning, customer contracts allowing variation of capacity, network optimization) can and will be integrated together into operator systems. These systems will automatically drive fast provisioning in the network. Standardization between vendors, between operators and between technologies will allow much simpler systems, which will translate into long term cost savings for operators.

In the short term, there is already widespread recognition of the value of signalling-based restoration in core transport networks. This can serve either to reduce "Out of hours" maintenance costs as a supplement to standard protection 
(replacing a backup path in the event of a failure, so as to allow extended time to repair) or to avoid deploying new capacity by using shared instead of dedicated protection.

A likely next step is the widespread use of "explicit routing" to implement provisioning decisions made by external systems. This (in conjunction with the maturing of standards for UNI and inter-vendor NNI) will allow a common process across a multi-vendor network, and increasingly support a "flow-through" management philosophy.

The biggest potential OPEX saving is where there are most trails to provision relatively low bandwidth circuits based on VC12-nv, VC4-nv, often supporting fractional Ethernet. Thus we expect to see this control-plane based provisioning gradually extend towards the network edge.

Clearly the extension to use distributed discovery and routing protocols is critically dependent on resolving the relationship with the network management systems.

\section{3. "NEXT-GENERATION SDH"}

Next-Generation SDH is not a precisely defined term. It refers to the new elements of flexibility in the SDH standards aimed to support data interfaces, standard data encapsulation and functionality supporting data aggregation. It is usually taken to include:

- Virtual Concatenation at VC12, VC3 and VC4 - building of "VCx-nv" Virtual Concatenation Groups (VCGs)

- LCAS (G.7042) allowing "hitless" variation of the size of a VCG.

- More highly integrated line-side xWDM I/F (and pluggable optical modules)

- Data interfaces (predominantly FastE and GigE, but also Fibre Channel, ESCON/FICON, and ATM). This trend includes increasing use of the "Ethernet in the First Mile" (EFM or IEEE802.3ah) standards for optical Ethernet with OAM.

- GFP (G.7041) encoding of these data interfaces into VCGs, both using delay-sensitive "transparent mode" and (more commonly) "frame mode".

- "Layer 2" data aggregation capability. Here there is considerable variation. Most commonly supported is the ability to create "Permanent Virtual Circuits" over Ethernet based on the use of VLAN tags to distinguish customer frame streams. This may or may not include "QinQ" - the ability to stack VLAN tags so as to preserve customer VLAN information. Also commonly included is some use of "MPLS draft Martini" based on provisioned MPLS labels. The traditional enterprise 
meaning of "Ethernet switching" (MAC address based learning bridges) is, in our view, of limited value in public networks (since MAC addresses cannot be summarized and spanning tree implies an acyclic network).

- Traffic management. Here again there are many variants. Policing of "fractional Ethernet" (subscribed bit rate < physical line rate) is fundamental. Concentration of "best effort" traffic is also appropriate; it saves hauling bits back to core routers just to throw them away. We do not believe that "frame relay-like" statistical multiplexing is appropriate in a transport device, since it greatly complicates the network and the service being offered may not be easily explained to a customer.

This collection of functionality provides attractive options for operators to optimize their transport networks in a mostly data environment. At the same time, it allows the preservation of the "carrier class" service qualities which are a major brand value for traditional operators, by providing guaranteed service and transport quality fast protection. In the short term, it will be provided by add-in cards extending the installed base. In our view, transport nodes in the medium term will be natively able to provide "permanent virtual circuit cross-connection" based on Ethernet and MPLS tags (and interworking with ATM). We are using the term "Frame Cross-Connect" (FXC) to describe this function.

These new data-oriented functions also imply new responsibilities for transport network management, which will need to represent end to end data services across transport paths (facilitating surveillance, and adding test capability to aid fault location and repair actions).

There has also been discussion of more extensive integration of IP functionality with transport ("Layer 3"). We are not currently persuaded of the value of this integration. Tying router functionality together with transport reduces the opportunity to scale both technologies independently. Moreover, it greatly increases the complexity of the device, and makes it substantially more complex to manage. Finally, it can get in the way of the Layer 3 interaction between the customer router and an IP service switch in the network, making the service less predictable (in particular concerning QOS). Our view is that network transport should be "the router's best friend" (providing managed aggregation and fast protection) and not a bad router. 


\section{ETHERNET IN THE TRANSPORT NETWORK}

\subsection{Transport}

Just as IP is becoming the dominant interface at the service level, we expect the same to be true for Ethernet at the physical level. Thus there is a clear case for many network services to be presented to customers as Ethernet. Some have argued from this premise that Ethernet should become the universal transport mechanism, "in order to avoid format conversions", and that this will lead to a substantial reduction in network cost. In our view, the comparison is not so clear. If we compare a "best effort" unprotected Ethernet transport network with statistical gain to a protected NG-SDH network offering guaranteed subscribed bit rates, then no doubt Ethernet is cheaper. However, we believe that operators do need to offer service guarantees in order to sustain their revenues.

In general, WAN transport does not have the same requirements as LAN. There are new requirements for OAM, guaranteed packet throughput, and latency in the WAN, and some LAN technologies (e.g. MAC address "learning bridges") do not scale appropriately. Therefore not all of the functions for WAN transport and aggregation can benefit from the high volumes of enterprise Ethernet components.

We believe that "native Ethernet" and "Ethernet over SDH" transport will coexist in the network, and have somewhat complementary roles.

In the access region, initiatives such as Ethernet in the First Mile (EFM) do allow for cost-effective Ethernet transport. We believe that these interfaces (over fibre and copper) will take a significant share of business access. At the same time, where TDM circuits still need to be carried, or protected access is required, Ethernet over SDH (GFP encoded to a Virtual Concatenation Group) is an effective solution.

Further into the network, requirements for protection and OAM are critical. Whilst there is certainly a role for "native" Gigabit Ethernet transport, we believe that the predominant mode of Ethernet transport will be GFP encoding over VC4nv or ODU bearers. Ethernet (GigE and 10GigE) will be widely used as a costeffective way of interconnecting network equipment inside buildings.

\subsection{Aggregation}

A key advantage of Ethernet as a physical interface is that the cost is not significantly affected by the physical line rate. Thus service providers have the opportunity to fit network termination equipment with substantial "headroom", and hence a long life without further visits to customer sites. However, most customers will not wish to pay for a full FastE or GigE through the network. Therefore there 
is a key role in access of policing these interfaces to the customer subscribed bit rate ("fractional Ethernet"). This subscribed rate needs to be flexible with relatively fine granularity.

A second advantage of high bandwidth Ethernet customer interfaces is the ability to multiplex several frame streams cost-effectively over a single broad interface. These "multiplexed Ethernet Private Line" services are commonly distinguished using VLAN tags. Transport nodes will need to preserve these "virtual circuit" labels across the network, so as to avoid using expensive IP routers to support basic private line services. They will also need to be able to interwork multiple virtual circuit technologies. So there is a need to map VLAN tagged frames to MPLS "draft Martini", and also to ATM VCs.

Hence transport nodes will need to evolve to support these "frame" technologies. At the same time, we do not see a good justification for a full IP/MPLS control plane, which would greatly complicate such node and especially its management. These services remain provisioned virtual circuits.

Since the services offered over these bearers are predominantly "new generation private line", the normal use requires guarantees of subscribed bit rate ("committed effort"). Transport nodes will also need to offer fair concentration of best effort traffic (allowing controlled overbooking of bearers for services such as Internet access).

\subsection{Interfacing to Routers}

As networks come to be mostly packet based, and the cost per bit of transport falls, there is a new opportunity for network optimization.

Current router networks use several levels of hierarchical concentration towards the core to reduce the total number of bits carried. We believe that (just as occurred in voice network digitalization in the 1980s) this router hierarchy will significantly flatten, and that there is a key opportunity to use transport nodes to reduce transit traffic through routers. Moreover, since transport node interfaces are a small fraction of the price of "carrier class" router cards of the same bandwidth, there is a role for the transit network in ensuring that router line cards are well filled.

Both on the access and the core side, a "de-layered" network of routers needs many more ports than it has physical line card slots. Therefore there is need for a flexible mechanism supporting multiple "virtual ports" on a single physical port, to support the core network scale which will be required. Moreover, the size of these "virtual routes" across the core will range widely (from 10s of Mbits/s to many Gbit/s).

There are several possible mechanisms to support this requirement. The most likely seem to be: 
1. Channelized line cards (e.g. STM-1 channels in STM-64). Currently using POS ("Packet over SONET") encoding - likely to move to GFP.

2. IP over ATM line cards (at rates up to STM-64).

3. GFP in (for example) STM-64 with use of the multiplexing field in the linear extension header to define frame streams, with transport nodes routing individual multiplex values to different VCGs (probably at VC4 granularity).

4. "Tagged Ethernet". Multiple frame streams (distinguished by VLAN tag or MPLS tunnel label) inside GigE or 10GigE.

The ATM option above will almost certainly be required, given the need to interwork with current large ATM networks, but probably does imply extra cost. The "GFP multiplexing" option is in some technical respects the most elegant, but relies upon the existence of appropriate transport layer functionality which is currently unavailable. Therefore, another option likely to be successful is "Tagged Ethernet", with an intelligent core transport node directing the different tagged streams to different VCGs (most likely VC4 granularity) and encoding with GFP.

[This implies functionality from the router, both in respect of per-LSP queuing and traffic management, and in respect of multiple routing adjacencies per physical interface.]

\section{MULTI-SERVICE ACCESS NODES}

Operators are discovering a real business need to make convergence happen. Reducing the number of edge platforms deployed in high volume in their networks is a major opportunity for OPEX reduction. They need to find new service revenues from added value on top of basic broadband and new services to small business. At the same time, they see the need to begin to plan their evolution to packet voice (even where no compelling short term reason for implementation exists).

In this context, "first generation" DSLAMs are being replaced by much more flexible multi-service Access nodes (MSANs), capable of terminating a wide variety of access technologies.

MSANs continue to support the DSLAM functionality, and hence require flexible ATM aggregation. They increasingly require to terminate Ethernet bearers over copper, fibre and even fixed wireless, and can also support Ethernet backhaul on the network side. They will support "combo" POTS/DSL line cards as well as packet voice gateways, allowing a graceful evolution from old to new voice networks without on-site intervention.

A key enabler for new service revenues is the ability to support "session-based services" - that is, usage-based charging for quality guarantees for particular 
content streams. A first step in this direction is the integration of the so called Local Access Concentrator (LAC) and (part of) Broadband Remote Access Server (B-RAS) functions [terminating PPP sessions from customers, initiating authentication, authorization and accounting (AAA) functions for the user (interacting with centralized network servers for these functions), and forwarding the traffic in appropriate "tunnels"]. There will also be a need for "media firewall" (flow identification and policing) functions for packet-based access to multiple services.

The predominant trend in current networks is for deployment of MSANs at copper distribution frames (collocated with existing switch remotes and DSLAMs). Many operators are also considering the possibility of street electronics, as an evolution bringing fibre nearer to the customer and allowing higher bandwidth (e.g. based on VDSL). This can be accommodated within an MSAN architecture, using small remote line shelves and aggregator nodes.

Finally, there is obviously scope for bringing together MSAN with edge transport functions (e.g. VC12 granularity SDH). The likely outcome is an architecture allowing for "remote line shelves" subtended from aggregation nodes.

\section{MPLS AND ATM}

Most profitable data services today are carried over ATM networks. Moreover, the most important new source of bandwidth in operator networks is residential broadband, which is aggregated using ATM. Hence "rumours of the death of ATM have been greatly exaggerated". Most operators have substantial and profitable ATM networks which they will not abandon quickly, and which they will require to evolve without service disruption. Whilst best effort IP traffic has huge volume, most such traffic does not support profitable services. Operators recognize that obtaining such profitability requires service delivery with appropriate and dependable quality. In the context of real attempts at network convergence including voice and video, differentiated QOS is completely essential.

[Note that Quality of Service is not completely assured by Class of Service technologies such as DiffServ. While DiffServ can ensure that some packets are treated with priority, it cannot ensure that there is sufficient capacity in the network to provide appropriate quality for the delivery of any particular service flow. This requires in addition the reservation of resources and admission control]

MPLS is intended to solve this problem by integrating a hierarchical "virtual circuit" technology with IP forwarding, so as to obtain a scalable IP control plane supporting QOS. As currently standardized, MPLS does not fulfill that promise completely. In particular, it does not support QOS-aware path selection beyond the boundaries of a single Internal Gateway Protocol (IGP) area, and it does not have 
adequate OAM capabilities. Scalable QOS support requires either changes to existing gateway protocols (IS/IS, OSPF) or the adoption of a scalable QOS-aware alternative (e.g. PNNI, which already addresses these problems for ATM networks).

We believe these problems can be solved, but they are not solved yet. Current generation routers are not appropriate and are probably not extensible to this functionality. We think, therefore, that MPLS is the most likely medium term solution to a converged core network, but that the necessary preconditions are not yet in place. When MPLS is deployed for that purpose, it will need to interwork effectively with the ATM installed base.

\section{WIRELESS ACCESS TECHNOLOGIES}

Wireless will be the predominant home networking technology, used not just for telephony but also for residential LANs and for distribution of multi-media.

Wireless LAN (IEEE 802.11x) technology will be widely deployed at "hot spots". There will be data solutions using roaming between $\mathrm{WiFi}$ and GPRS/UMTS, and wireless connected (e.g. Bluetooth) voice solutions allowing roaming between the fixed and mobile networks. Since WiFi will be deployed by many small access providers (shopping malls etc.) there will be sharing of backhaul infrastructure between many networks and technologies. Extensions to 802.11 allowing extended point-point connectivity, as well as new technologies such as IEEE 802.16, will be used as "fixed wireless", and will be integrated into MSANs.

In total, these new technologies will eat into the revenues expected of UMTS. However, this will remain the network with the largest coverage used for high volume mobile telephony.

\section{MULTIMEDIA SOFTSWITCHES}

Packet voice technology has been "emerging" for a long time in public networks. Whilst there has been substantial deployment of IP PBXs, most operators have not made major investments in public network systems. There are several reasons for this:

1. Existing voice systems are stable, dependable and do not require major new investment

2. Base fixed voice demand is not growing. There has been growth (in particular because of dial IP access) but this has been addressed where 
necessary by specialized bypass solutions, and is now disappearing because of broadband substitution.

3. Whilst basic packet voice technology is not in doubt, there are still major concerns about quality, security and scalability for the public network, and the evolution issues are complex.

In our view, this situation is now changing. Operators are beginning to have real obsolescence concerns about their voice infrastructure, and they recognize that any replacement strategy will take some time to deploy. Moreover, they are now convinced that they cannot survive on their traditional revenues, and therefore need a flexible service architecture.

The challenge in designing a public network packet voice solution is to balance several issues:

a. Internet-derived technologies such as SIP give great flexibility for rapid deployment of new services. Operators must be able to support new kinds of services and new media if they are to invest in a whole new service infrastructure.

b. A totally "transparent" IP network gives operators no opportunity to provide appropriate quality and dependability for a public network offering, and no way of charging for their services.

c. A valuable service depends on universal inter-operability with fixed and mobile networks, and hence must interface to current standards and feature sets, and support current regulatory and government requirements.

Once again, we believe that the technology is now becoming sufficiently mature to address these concerns. State of the art softswitch systems consist of a set of components connected together by standardized interfaces. They offer:

- controlled access to resource (and other networks) based on "media firewalls", which police and translate between multiple IP address spaces and also manage QOS for media streams on the boundaries of an operator IP domain

- a full range of gateway protocols including MGCP, H.323 and H.248.

- controlled access to SIP servers and devices by way of SIP servers, gateways, and SIP-T tunneling of PSTN protocols.

- Full interoperability with existing network features and protocols

- Flexible service intelligence based on "new generation IN"-in particular, based on Parlay

- Service interworking with $2.5 \mathrm{G}$ and $3 \mathrm{G}$ mobile networks using CAMEL \& Parlay

- High dependability and network wide fault tolerance,

- Flexibility in size and location, allowing deployment at sites convenient to the operator (typically a small number of centralized locations) 


\section{OSS STANDARDS AND PROCESSES}

As a result of network, technology and service evolution most operators run a number of separate business addressing different service needs. Organizational responsibilities are partitioned in order to give focus and enable effective decisionmaking. Even within an individual business there are typically several types of network and these may be partitioned in different ways organizationally and will use equipment from multiple vendors. The operational support systems reflect the organizational boundaries, resulting in multiple management systems that overlap in functionality, and have been interconnected in an ad hoc manner. In recognition of the inefficiencies inherent in this pragmatic method of developing OSSs, many operators are attempting to move to an approach based on operational process modelling, on the basis that organizations can change but the underlying business processes are stable.

In addition there are a number of industry initiatives aimed at better standardization of the techniques and terminology used to define operational support systems. There are independent software vendors who are developing components for the next generation of OSS including platforms to support business to business interfaces, web based user interfaces, and distributed applications, as well as the applications themselves.

We believe that there will be a general trend towards deployment of next generation OSS architecture supporting a component-based approach. But there are significant organizational barriers to be overcome and significant expenditure to fund. Operators will take advantage of these developments depending on their own internal priorities and they will fall into two broad categories. Firstly, the large majority of OSS changes will be those that can show a positive business benefit in less than a year. They will be targeted at operational cost saving largely in an existing network context. [Major OSS changes de-coupled from network infrastructure changes often result in the need for complex migration strategies to ensure continuity of service using legacy management interfaces]. Secondly, there will be strategic investments that will be combined with significant changes to networks. These will involve organizational change with a view to major restructuring of the business cost base and adding flexibility to deliver new services. Here again there may be the issue of migration of crucial service specific data and functionality if the new network platform has to deliver existing services.

In the telecommunications industry two standards cultures exist, the ITU-T and the IETF. Associated with these there are two fundamentally different approaches to management interfaces at the equipment level. These are the ISO protocol based Q3/Qx from the ITU-T and the IP protocol based SNMP from the IETF. IP equipment often also uses proprietary command line interfaces (CLI) and web browser style interfaces (HTTP based) while many different proprietary interfaces 
exist on traditional telco equipment. Most network operators have implementations that use both types of interface as they have both transport (ITU-T) and packet (IETF) based networks. If we assume that IP/MPLS is the de facto protocol for the core of future networks then it is likely that the de facto management interface will be SNMP. Because of the security shortcomings of SNMP v1 and v2 a migration to SNMP v3 seems probable (and may be mandated by some influential customers).

There are drives towards process and architecture commonality championed by the TeleManagement Forum's NGOSS initiative. NGOSS (New Generation Operations Systems and Software) is a set of guidelines for the industry to build software in a more structured and complementary way than has been done historically.

- a "loosely coupled" distributed component architecture

- along with functioning application components

- the components interact through a common information bus

- the components can be programmed through the use of a process management tool to control the business processes of the service provider using the functionality provided by the components

Due to the cost of change, all these interface styles are likely to persist for a long time. Thus there will be an ongoing requirement for multiple mediation functions in an integrated management system. This is another reason why significant change in the OSS of an established operator generally needs to be coupled with significant change in the equipment layer when the consideration of legacy interfaces can be minimized.

\subsection{OSS Technology evolution}

A number of technology threads will have significant influence on OSS implementations.

The general development of computing and storage technology will allow more complex functions to be performed in near real time and will allow more functionality to be deployed on the elements themselves. [Many of the complexities of existing element management applications arise from constraints on processing power in equipment controllers and DCN bandwidth].

The development of more robust and functional distributed application environments, web services and better information modelling techniques supported by tools is enabling faster implementation and more automation of operational processes. For example, the combination of Java-based mobile code frameworks and environments supporting a "component lifecycle" model allow standard ways of deploying new functionality and retiring old. 


\section{CONCLUSIONS}

The emergence of improved processing frameworks and information modeling tools is having a significant impact on the time to develop new OSS applications, but to date no one has found a simple way to migrate from existing to new systems. All such processes require some form of encapsulation concept and the implementation of an adaptation function, which may be able to be retired when the legacy system is replaced.

Telecommunication networks and services will need to evolve on order to allow for a more efficient delivery of existing and new broadband services, whilst facing a significant decrease in revenue per transported and switched bits.

As broadband new services require more bandwidth and a more complex delivery, so network complexity and global capacity is growing fast while revenues per bit are falling.

Operator face a difficult task requiring on one hand to converge their networks in order to achieve better cost efficiency, whilst at the same time making sure that considerable network scalability is permitted in order to cope with the growing demand.

Several technologies are however available to meet this challenge and allow for an evolutionary network migration. This article provides a brief overview of the most significant, which are most likely to be employed in the making of the next generation networks 